\title{
Topological and Shape Optimization of Flexure Hinges for Designing Compliant Mechanisms Using the Level Set Method
}

\author{
Benliang Zhu ${ }^{1 *} \mathbb{D}$, Xianmin Zhang ${ }^{1}$, Min Liu ${ }^{2}$, Qi Chen ${ }^{1}$ and Hai Li ${ }^{1}$
}

\begin{abstract}
A flexure hinge is a major component in designing compliant mechanisms that offers unique possibilities in a wide range of application fields in which high positioning accuracy is required. Although various flexure hinges with different configurations have been successively proposed, they are often designed based on designers' experiences and inspirations. This study presents a systematic method for topological optimization of flexure hinges by using the level set method. Optimization formulations are developed by considering the functional requirements and geometrical constraints of flexure hinges. The functional requirements are first constructed by maximizing the compliance in the desired direction while minimizing the compliances in the other directions. The weighting sum method is used to construct an objective function in which a self-adjust method is used to set the weighting factors. A constraint on the symmetry of the obtained configuration is developed. Several numerical examples are presented to demonstrate the validity of the proposed method. The obtained results reveal that the design of a flexure hinge starting from the topology level can yield more choices for compliant mechanism design and obtain better designs that achieve higher performance.
\end{abstract}

Keywords: Topology optimization, Compliant mechanisms, Flexure hinges, Level set method

\section{Introduction}

Compliant mechanisms have been widely used in precision engineering applications in which precision of motion, compactness, and feasibility are required. Examples can be easily found in, for example, micro- and nano-manipulation and manufacturing [1-3] and optical alignment $[4,5]$. Compliant mechanisms often refer to monolithic or jointless structures that transfer an input force or displacement to another point through elastic body deformation [6, 7]. For example, in a microelectro-mechanical system (MEMS), movement between adjacent members is nearly entirely achieved through flexure hinges. Therefore, to design compliant mechanisms, a flexure hinge is regarded as a key component $[8$, 9]. Unlike the rigid mechanical connections that allow

\footnotetext{
*Correspondence: meblzhu@scut.edu.cn

${ }^{1}$ Guangdong Provincial Key Laboratory of Precision Equipment and Manufacturing Technology, South China University of Technology, Guangzhou 510640, China

Full list of author information is available at the end of the article
}

different kinematic degrees of freedom (DOFs), a flexure hinge can only provide a limited relative motion between two rigid parts, as illustrated in Figure 1(a), (b).

Since Paros and Weisbord [10] proposed right circular flexure hinges and introduced an analytical approach to derive exact and approximate compliance equations, substantial research has been spent on creating flexure hinges having new configurations that achieve high performance [11-14]. For instance, a hybrid flexure hinge, which consists of half a hyperbolic flexure hinge and half a corner-filleted flexure hinge, was developed in Ref. [15]. Comparison studies with five kinds of common notch flexure hinges have also been conducted quantitatively with respect to compliance, precision, compliance precision ratios, and maximum stress. In addition, right-elliptical, corner-filleted, V-shaped and cycloidal, and polynomial-type flexure hinges, as well as those with conic sections, have also been developed [12, 16, 17].

However, although many new configurations of flexure hinges have been proposed, the design procedures for 

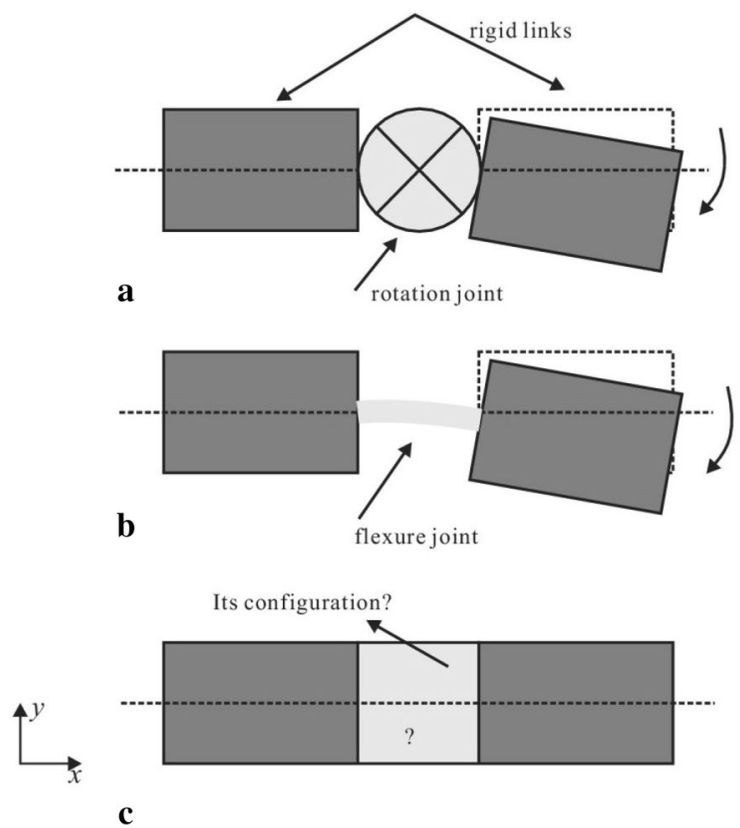

Figure 1 Motivation of this study: a Two rigid links are connected by a traditional rotation joint. b Two rigid links are connected by a flexure hinge. $\mathbf{c}$ What should be the configuration of the flexure hinge?

these configurations are often intuitive and thus depend to a large extent on designers' individual intuition, experience, and inspiration. For conceptually designing flexible joints, screw theory-based methods have been developed [18-21]. However, these types of methods are often adopted when designing compound-type flexure hinges. Recently, structural topology optimization methods have also been adopted for designing configurations of flexure hinges. Examples of using these methods can be found in Refs. [22-26]. The unique feature of the topology optimization method is that the optimal solution can be generated automatically even though the topology of the mechanism is not known in advance. Since Bendsoe and Kikuchi developed the homogenization method [27], topology optimization has been exhaustively explored in both theoretical studies and practical applications [2831]. Recently developed topology optimization methods include density-based approaches, such as the solid isotropic material with penalization (SIMP) [31-33] and evolutionary structural optimization (ESO) [34, 35] as well as the level set method [36, 37]. In contrast to density-based methods, the level set method can avoid ambiguities of intermediate material phases (i.e., provide a smooth boundary of the designed structures [38]).

In using topology optimization methods to design flexure hinges, the first task it to devise an optimization formulation. Several formulations have been established as in Refs. [22, 39]. The objective is to maximize the compliance in the desired direction while minimizing the compliances in the other directions. In addition, a constraint to obtain the accuracy of the revolution movement is also used. However, the obtained flexure hinges seem asymmetric with respect to the longitudinal axis [12]. This increases the difficulty of real-world applications of the obtained flexure hinges.

Therefore, in this study, we present a systematic method for designing revolute flexure hinges by considering the symmetric constraint. An optimization model is developed by considering the functional requirements of the flexure hinge (i.e., maximizing the compliance in the desired direction while minimizing the compliances in the other directions). The weighting sum method is used to construct an objective function in which a selfadjust method is used to set the weighting factors. A constraint on the symmetry of the obtained configuration is developed.

The remainder of the paper is organized as follows. In Section 2, a level set method that does not require re-initialization is introduced. In Section 3, optimization formulations are developed for the topology optimization of flexure hinges by considering an asymmetric constraint. In Section 4, sensitivity analysis and an optimization algorithm are introduced. In Section 5, several numerical examples are presented to demonstrate the validity of the proposed method. A conclusion is provided in Section 6.

\section{Level Set Method}

The underlying idea behind the level-set-based topology optimization is that the structural layout is defined by a level set function $\phi$ as:

$$
\begin{cases}\phi(x, t)>0, & \text { if } \boldsymbol{x} \in \Omega, \\ \phi(\boldsymbol{x}, t)=0, & \text { if } \boldsymbol{x} \in \Gamma, \\ \phi(\boldsymbol{x}, t)<0, & \text { if } \boldsymbol{x} \in D \backslash \Omega,\end{cases}
$$

where $D$ is the design domain that completely contains the material domain $\Omega, D \backslash \Omega$ is the void area, $\Gamma$ is the structural boundary, $x$ is a point in the design domain, and $t$ is the pseudo-time.

The optimization process is described by the evolution of the level set function governed by [37]:

$$
\frac{\partial \phi}{\partial t}+V_{n}|\nabla \phi|=0
$$

where $V_{n}$ denotes the velocity field that determines the motion of the interface. During the optimization process, the level set function gradients at the design interface must be controlled to ensure stability. This is often achieved by periodically re-initializing the level set function to a signed distance function, which is known as the re-initialization procedure [40]. However, 
re-initialization can slightly move the zero level set away from the expected position and introduce some fundamental problems (e.g., when and how to perform the re-initialization) that have yet to be solved [36]. To overcome these problems, a generalized Hamilton-Jacobi equation was developed in Ref. [41]. The idea is to replace Eq. (2) to update the level set function by using the following equation:

$$
\frac{\partial \phi}{\partial t}+V_{n}+\omega \nabla \cdot\left(d_{r}(|\nabla \phi|) \nabla \phi\right)=0,
$$

where $\nabla \cdot\left(d_{r}(|\nabla \phi|) \nabla \phi\right)$ is the diffusion part and $\omega$ is a predefined parameter [41]. The diffusive rate $d_{r}(|\nabla \phi|)$ is defined as:

$$
d_{r}(|\nabla \phi|)=(|\nabla \phi|-1)(2|\nabla \phi|-1) .
$$

The added diffusion can maintain the level set function at a location close to a signed distance function near the structural boundaries while also forcing the level set function to be a constant at locations far removed from the structural boundaries. For additional details, please refer to Ref. [41].

\section{Design Formulations}

We developed a practical formulation for the topological synthesis of flexure hinges. A general design domain for topology optimization of flexure hinges is illustrated in Figure 2, where $\boldsymbol{D}$ is the design domain and $\boldsymbol{R}$ is the adjacent rigid member. The designed configuration is for planar applications. This means that the two adjacent rigid members are expected to experience limited relative rotation about the sensitive axis, which is an axis that is perpendicular to the $x y$ plane (Figure 1). Figure 2(a) indicates the boundary conditions for a generic flexure hinge. The design domain $\boldsymbol{D}$ is fixed at the boundary $\Gamma_{d}$. Note that this boundary condition is valid because the relative movement of two members can be replicated by considering that one is fixed. The width $L$ of the rigid member $R$ is set to be at least equal to or greater than the width $l$ of the design domain $\boldsymbol{D}$. The study in Ref. [42] suggests that forces should be applied at a point where at least $L \geq 3 l$. Therefore, in this study, $L=3 l$ is chosen.

\subsection{Objectives}

The capacity of rotation is the critical factor in characterizing a flexure hinge. The rotation requirement means that a structure must be found by using the given limit of material that can fulfill a finite rotation around the axis that is perpendicular to the $x y$ plane when the rigid body $\boldsymbol{R}$ is applied on a force $F_{y}$ at point 1, as shown in Figure 2(a). Suppose that the displacement at point 1 due to $F_{y}$ is indicated as $u_{1 y}$. The design goal can be accomplished by maximizing $u_{1 y}$.

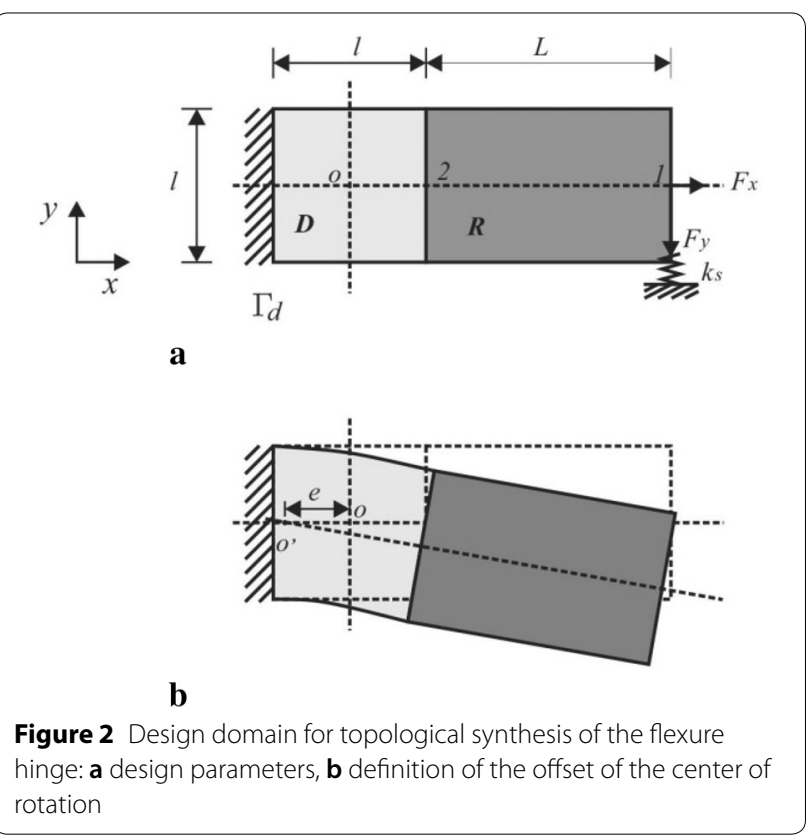

Note that the problem of maximizing $u_{1 y}$ has a trivial solution. One can, in principle, obtain an infinitely large displacement by removing the whole material in the design domain $D$. In this study, to overcome this problem, we attach an artificial spring with stiffness of $k_{s}$ to point 1 to limit the magnitude of $u_{1 y}$. In addition, a stiffness requirement should be imposed on the designed flexure hinge. As mentioned in Ref. [22], an ideal revolute flexure hinge should prevent movement in other directions. This can be accomplished by minimizing the horizontal displacement $u_{1 x}$ of point 1 resulting from vertical load $F_{x}$, as shown in Figure 2(a).

Therefore, a design problem with two objectives is considered. The easiest means of dealing with this problem is to use the weighting sum method to transfer it into an optimization problem with a single objective. Therefore, the objective for topology optimization of revolute flexure hinges can be expressed as:

$\min :-u_{1 y}+\alpha u_{1 x}$,

where $\alpha$ is the weighting factor.

One major issue of the weighting sum method is that the value of the weighting factor must be preset. Normally, no uniform means exists to determine its value, and a different design problem may require different values. To overcome this problem, we adopt the self-adjust scheme proposed in Ref. [43]. The underlying idea is to update the values of the weighting factors during each iteration step by using the following equation:

$$
\alpha^{k+1}=\frac{u_{1 x}^{k}}{u_{1 y}^{k}},
$$


where $\alpha^{k}$ is the weighting factor that changes with each iteration $k$ of the optimization algorithm, and $u_{1 x}^{k}$ and $u_{1 y}^{k}$ denote the values of $u_{1 x}$ and $u_{1 y}$ in the $k$ th iteration, respectively. For the first iteration, one can simply set $\alpha$ to 0 . We set the weighting factors in this manner because in iteration $k+1$, if $u_{1 x}^{k}$ becomes larger than that of the last iteration, whereas $u_{1 y}^{k}$ becomes smaller, meaning that the obtained structure becomes unsteady (i.e., the tendency of generating a disconnected structure), then $\alpha^{k+1}$ will increase. This in turn will increase the weight of $u_{1 x}$, causing the created structure to become much stiffer.

\subsection{Constraints}

In the design of the topology of a flexure hinge, we are interested in the optimal placement of a given elastic material inside $\boldsymbol{D}$. In other words, we should determine those points that should be material points and those that remain void. The limited given elastic material leads to the following maximum material usage constraint:

$$
V o l=\int_{D} H(\phi) \mathrm{d} \Omega \leq[V o l],
$$

where the maximal material usage is constrained with an upper limit $[\mathrm{Vol}] . H(\phi)$ is the Heaviside function defined as:

$$
H(\phi)=\left\{\begin{array}{l}
1, \text { if } \phi>0 \\
0, \text { if } \phi \leq 0 .
\end{array}\right.
$$

Another important factor in characterizing a flexure hinge is the precision of the rotation (i.e., the center of rotation [16]). For real-world applications, an ideal revolute hinge can cause the center of the rotation to be fixed at point $o$, as shown in Figure 2(b). We define a parameter $e$ as the offset of the center of rotation. Then, to realize a high precision of rotation, $e$ should be as small as possible. Therefore, for a small rotation angle, the following condition should be satisfied:

$$
e^{2}=\left(\frac{u_{2 y}}{u_{1 y}-u_{2 y}} L-\frac{l}{2}\right)^{2} \leq \zeta
$$

where $u_{2 y}$ is the vertical displacement at point 2 due to the load $F_{y}$ and $\zeta$ is a positive small constant.

The last criterion for topology optimization of flexure hinges concerns their geometric symmetry. As shown in Figure 3, flexure hinges can be identified by their symmetry about the transverse $(y)$ or longitudinal $(x)$ axis. A fully symmetric flexure hinge can be realized if it is symmetric around both the $x$ and $y$ axes. For a single-axis flexure hinge, four cases are possible with respect to the reference domain, as indicated in Figure 3. For the first case, the upper left quarter of the design domain is taken as the

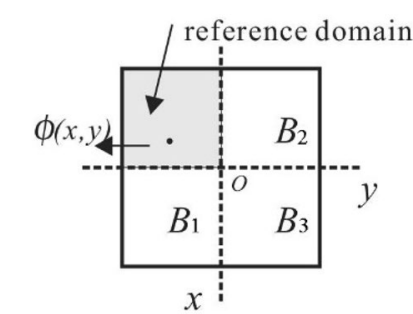

a
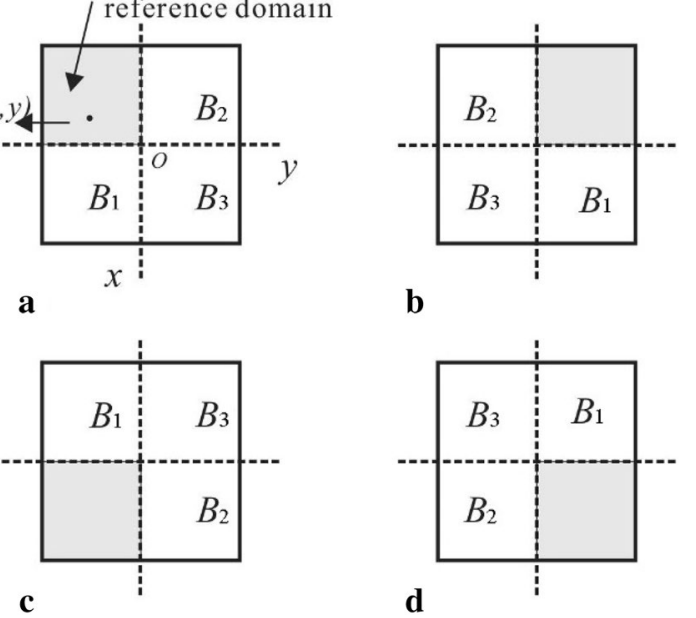

b

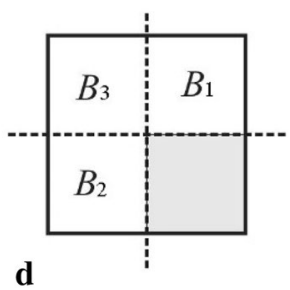

Figure 3 Possible cases for determining the references domain: a case $1, \mathbf{b}$ case $2, \mathbf{c}$ case 3 , and $\mathbf{d}$ case 4

reference domain. Then, the level set function in the other three quarters is determined by using the following three equations:

$$
\begin{array}{ll}
B_{1}: & \phi_{x}=\phi(-x, y), \\
B_{2}: & \phi_{x}=\phi(x,-y), \\
B_{3}: & \phi_{x}=\phi(-x,-y),
\end{array}
$$

where $\phi(x, y)$ is the level set function value at point $(x, y)$ in the reference domain and $\phi_{x}$ is the level set function value of point $x$ in the domain of $B_{1}, B_{2}$ or $B_{3}$. We can easily derive the setting rules of the other three cases.

\subsection{Optimization Model}

Incorporating the level set method, a possible formulation for topology optimization of the flexure hinges can be formulated as follows:

$\min : \quad J(u, \phi)=\alpha u_{1 x}(u, \phi)-u_{1 y}(u, \phi)$,

s.t., $\quad(u, \phi)=\left(\frac{u_{2 y}(u, \phi)}{u_{1 y}(u, \phi)-u_{2 y}(u, \phi)}-\frac{1}{6}\right)^{2} \leq \zeta$,

$V o l=\int_{D} H(\phi) \mathrm{d} \Omega \leq[V o l]$,

$B_{1}: \phi_{x}=\phi(-x, y)$,

$B_{2}: \quad \phi_{x}=\phi(x,-y)$,

$B_{3}: \quad \phi_{x}=\phi(-x,-y)$,

$a(u, v, \phi)=l(v, \phi), \quad \forall v \in U$, 
where $u$ is the state variable, $U$ denotes the space of kinematically admissible displacement fields, and $v$ denotes the arbitrary virtual displacement in the space $U$. The energy bilinear functional $a(u, v, \phi)$ and the load linear functional $l(v, \phi)$ are respectively expressed as:

$$
\begin{aligned}
& a(u, v, \phi)=\int_{D} E_{i j k l} \varepsilon_{i j}(u) \varepsilon_{k l}(u) H(\phi) \mathrm{d} \Omega, \\
& l(v, \phi)=\int_{D} f v \delta(\phi)|\nabla \phi| \mathrm{d} \Omega,
\end{aligned}
$$

where $E_{i j k l}$ and $\varepsilon_{i j}$ are the elasticity tensor and strain tensor, respectively. Because the design condition considered in this study does not concern the body force, only the boundary traction $f$ is considered in the previous equations, where $\delta(\phi)$ is the Dirac delta function defined as:

$$
\delta(\phi)=\frac{\partial H(\phi)}{\partial \phi} .
$$

\section{Sensitivity Analysis and Optimization Algorithm}

To use the level set method, finding an appropriate velocity $V_{n}$ is essential. A common technique is to solve the constrained optimization problem by constructing an augmented objective functional as:

$$
\begin{aligned}
L(u, \phi)= & J(u, \phi) \\
& +\lambda_{1}\left(\int_{D} H(\phi) \mathrm{d} \Omega-[V o l]\right)+\lambda_{2}(e(u, \phi)-\zeta),
\end{aligned}
$$

where $\lambda_{1}$ and $\lambda_{2}$ are Lagrange multipliers. The shape gradient of the general objective function $L$ can be determined effectively as:

$$
\frac{\partial L(u, \phi)}{\partial t}=\int_{\Gamma} G V_{n} \mathrm{~d} \Gamma,
$$

where $G$ is known as the shape gradient density [44]. To find the optimized structure, we can simply take $V_{n}=-G$. This is the common strategy for the level set method in structural optimization [36].

For the problem considered in this study, we have:

$$
\frac{\partial L(u, \phi)}{\partial t}=\frac{\partial J(u, \phi)}{\partial t}+\lambda_{1} \frac{\partial \int_{D} H(\phi) \mathrm{d} \Omega}{\partial t}+\lambda_{2} \frac{\partial e}{\partial t},
$$

where

$$
\begin{aligned}
& \frac{\partial J}{\partial t}=\frac{\partial u_{1 x}}{\partial t}+\alpha \frac{\partial u_{1 y}}{\partial t} \\
& \frac{\partial \int_{D} H(\phi) \mathrm{d} \Omega}{\partial t}=\int_{D} \frac{\partial H(\phi)}{\partial t} \mathrm{~d} \Omega=-\int_{D} \delta(\phi) V_{n}|\nabla \phi| \mathrm{d} \Omega,
\end{aligned}
$$

$$
\frac{\partial e}{\partial t}=2\left(\frac{u_{2 y}}{u_{1 y}-u_{2 y}}-\frac{1}{6}\right) \frac{u_{1 y} \frac{\partial u_{2 y}}{\partial t}-u_{2 y} \frac{\partial u_{1 y}}{\partial t}}{\left(u_{1 y}-u_{2 y}\right)^{2}} .
$$

We can see that solving the shape derivative of $L$ can be transferred into solving $\frac{\partial u_{11}}{\partial t}, \frac{\partial u_{1 y}}{\partial t}$, and $\frac{\partial u_{2 y}}{\partial t}$. By considering the boundary conditions of the considered problem and using the shape sensitivity analysis [45], the shape sensitivities of $u_{1 x}, u_{1 y}$, and $u_{2 y}$ can be obtained respectively as:

$$
\begin{aligned}
& \frac{\partial u_{1 x}}{\partial t}=\int_{\Gamma_{D}} E_{i j k l} \varepsilon_{i j}\left(u_{1}\right) \varepsilon_{i j}\left(u_{1}\right) V_{n} \cdot n_{0} \mathrm{~d} \Gamma, \\
& \frac{\partial u_{1 y}}{\partial t}=\int_{\Gamma_{D}} E_{i j k l} \varepsilon_{i j}\left(u_{2}\right) \varepsilon_{i j}\left(u_{2}\right) V_{n} \cdot n_{0} \mathrm{~d} \Gamma, \\
& \frac{\partial u_{2 y}}{\partial t}=-\int_{\Gamma_{D}} E_{i j k l} \varepsilon_{i j}\left(u_{2}\right) \varepsilon_{i j}\left(u_{3}\right) V_{n} \cdot n_{0} \mathrm{~d} \Gamma,
\end{aligned}
$$

where $n_{0}$ is the normal unit vector to $\partial \Omega, u_{1}$ and $u_{2}$ denote the displacement fields caused by the load of $F_{x}$ and $F_{y}$, respectively, and $u_{3}$ is the structural displacement field induced by applying only a unit dummy load at point 2 (see Figure 2). By substituting Eqs. (26)-(31) into Eq. (25), we can obtain the shape sensitivity of the Lagrange formulation $L$. For more details on the sensitivity analysis, please refer to Refs. [37, 45].

Note that the geometry constraints B1, B2, and B3 can be easily realized by directly operating the level set function $\phi$. Considering their derivative information is not required. Therefore, a possible algorithm for solving the topology optimization of flexure hinges can be stated as in Algorithm 1.

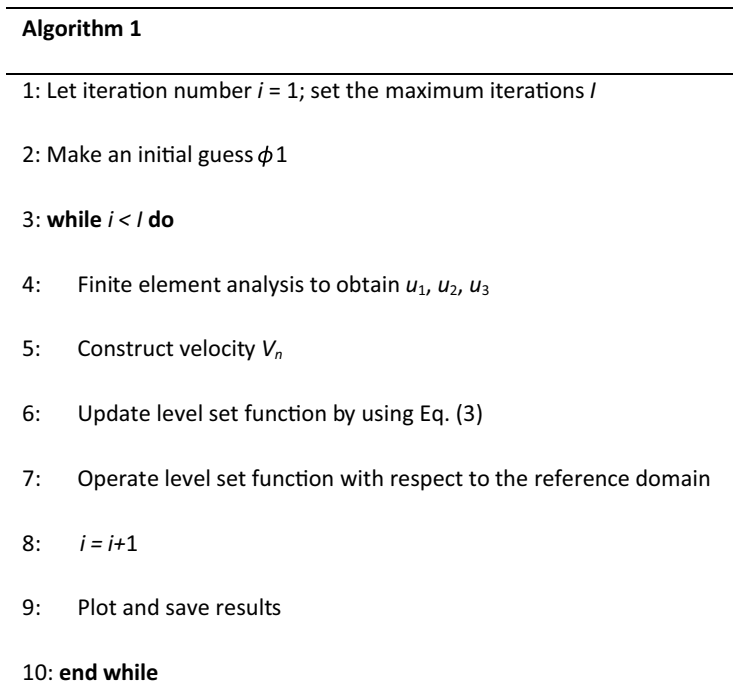




\section{Results and Discussion}

In this section, the numerical results for topology optimization of flexure hinges using the proposed method are presented. For the following numerical cases, the artificial material properties are described as Young's modulus for solid material $E$ is $1 \mathrm{GPa}$ and Poissons ratio $v$ is 0.3 . The input loads $F_{x}$ and $F_{y}$ are both set to $1 \mathrm{mN}$. The design domain $D$ is discretized by using $50 \times 50$ bilinear quadrilateral elements, and the rigid member $R$ is discretized by using $50 \times 150$ bilinear quadrilateral elements for elastic analysis. The size of the element is $1 \times 1 \mathrm{~mm}$.

\subsection{General Case}

For the first example, we set $k_{s}=0.02 \mathrm{~N} / \mathrm{m}$. The maximum material usage is set to $20 \%$ of the total material that can occupy the whole design domain $\boldsymbol{D}$. The upper limit of the offset $e$ is set to 0.01 . To implement the proposed method, new holes cannot be freely created because of the lack of an inherent hole-nucleation scheme. Therefore, a design with a relatively complicated topology (containing numerous holes) is used as the initial configuration, as shown in Figure 4(a). Note that for this studied case, the reference domain is chosen according to Figure 3(a).

The optimization process ran for 80 iterations. Some intermediate configurations and the final topology of the optimized flexure hinge are shown in Figure 4.
Remarkable shape and topological changes occurred at the first 20 iterations of the optimization process. For the last 60 iterations, the overall topology showed little change, although geometrical adjustments still had to be addressed to approach the optimal region. This indicates that the proposed method was computationally effective. The optimized flexure hinge is similar to that of the cartwheel flexure hinge, which is often regarded as a flexure hinge with ultra-high rotation accuracy.

The convergence histories of $u_{1 y}, u_{1 x}$, volume ratio, and the offset e are shown in Figure 5. The convergence history of the weighting factor $\alpha$ is shown in Figure 6. We can see that by adding a spring with $k_{s}=0.02 \mathrm{~N} / \mathrm{m}$, the magnitude of $u_{1 y}$ is restrained within $50 \mu \mathrm{m}$. Figure 6 reveals that with the shape and topological change during the optimization process, the weighting factor can adjust its value to ensure a well-posed design. This can overcome the difficulty of setting the value of the weighting factor artificially.

\subsection{Effect of the Reference Domain}

We next investigated the effects of the reference domain on the topology results. All parameters were set to the same as those given in Section 5.1. Similarly, an initial configuration like that shown in Figure 4(a) was used for all studied cases.

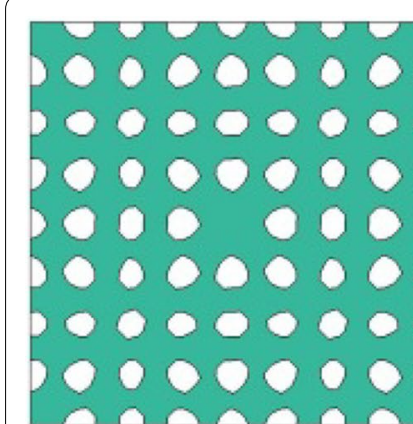

$\mathbf{a}$



e

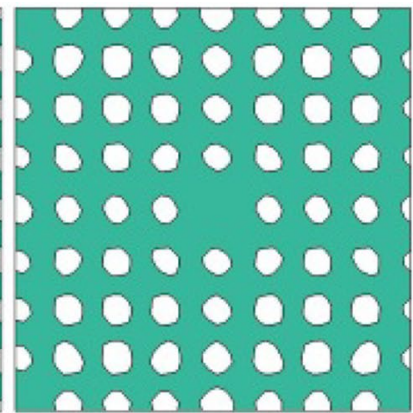

b

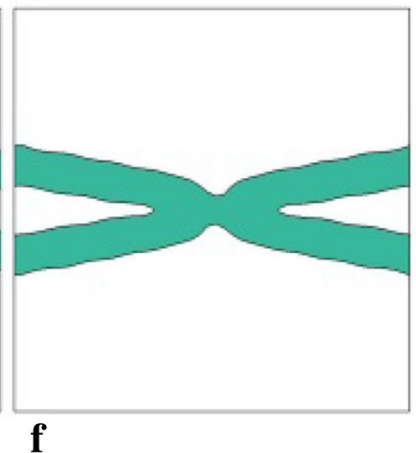

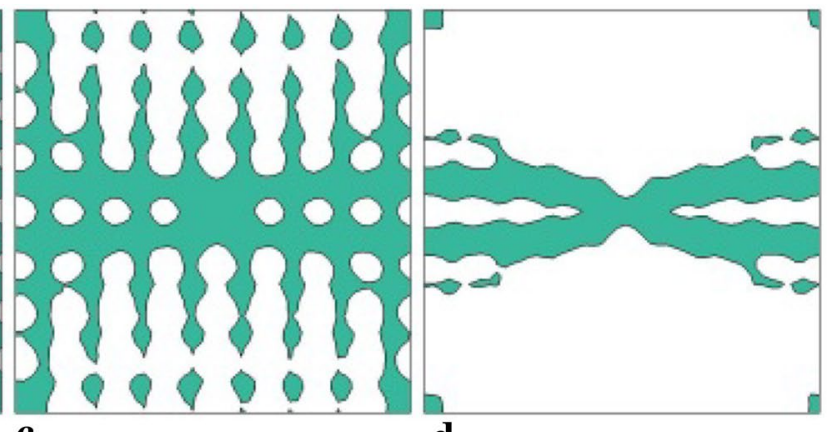

c

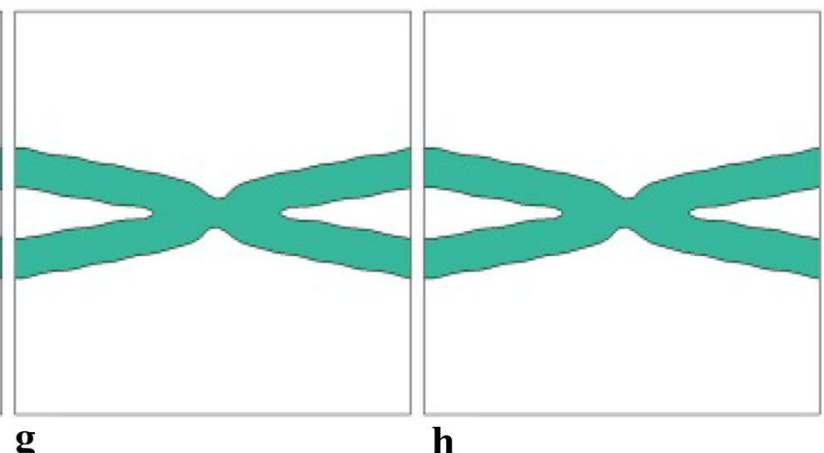

Figure 4 Initial configuration, intermediate designs, and the final topology of the flexure hinge: a initial configuration, b step 1, c step 5, d step 10, e step 20, $\mathbf{f}$ step 40, $\mathbf{g}$ step 60, and $\mathbf{h}$ step 80 (final) 

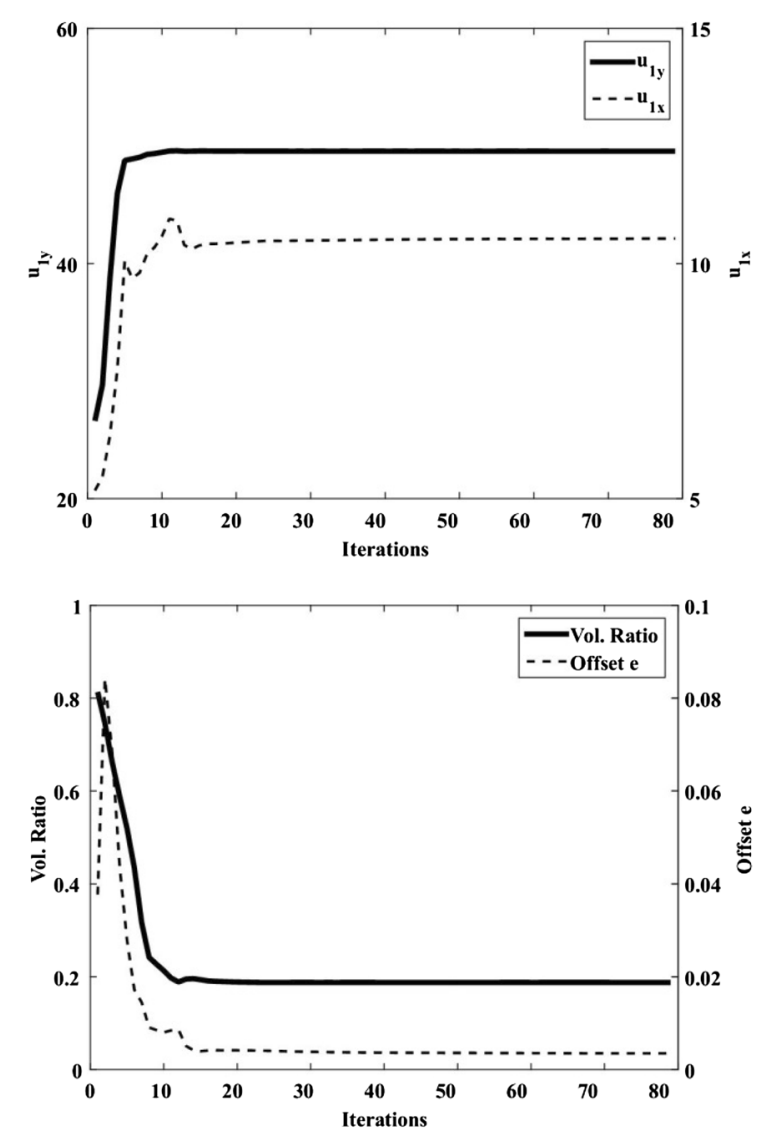

Figure 5 Convergence histories of $u_{1 y}(\mu \mathrm{m}), u_{1 x}(\mu \mathrm{m})$, volume ratio, and the offset $e$

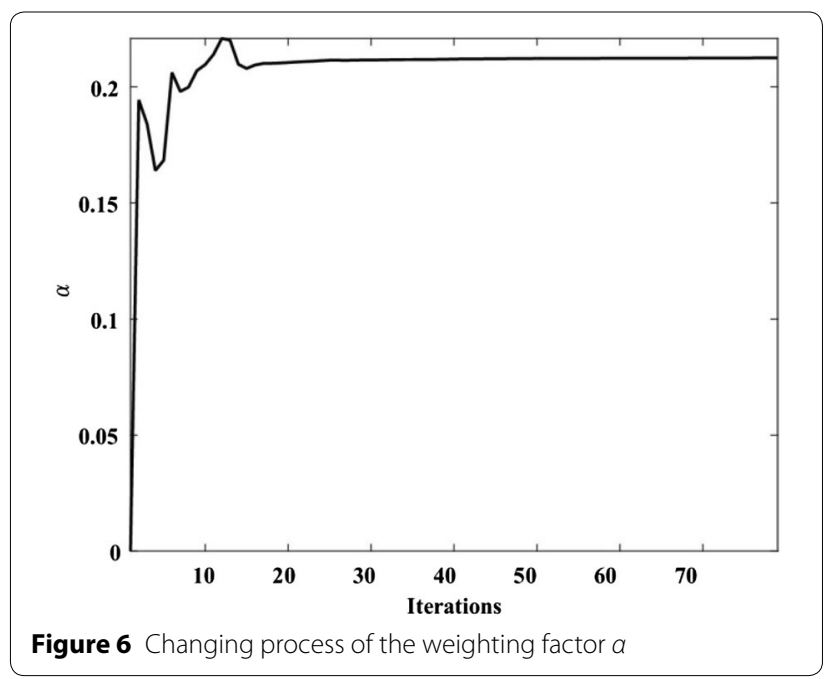

Four cases were studied, including one that did not consider the symmetry constraint and three possible reference domains that are shown in Figure 3(a)-(c). The corresponding optimized flexure hinges are shown in Figure 7. These results are also quantitatively compared with one another in Table 1.

Figure 7(a) shows that the optimized flexure hinge is indeed always symmetrical with the longitudinal axis $x$ even though no symmetry constraint is considered. This can also be seen in Figure 4(h) and Figure 7(b)-(d). Results indicate that choosing the reference domain with respect to the transverse axis $y$ is essential to obtaining a meaningful design. When we chose the reference domain according to Figure 3(b) and (d), the obtained flexure hinges are shown in Figure 7(c) and (d), respectively. Both obtained flexure hinges had a large offset $e$ (i.e., a lower rotational accuracy), as indicated in Table 1.

\subsection{Effect of the $k_{s}$}

We further investigated the effects of the output spring $k_{s}$ on the topology results of flexure hinges. Several cases with different output spring stiffness $k_{s}$ were analyzed. The maximum material usage was set to $20 \%$ of the total material that could occupy the whole design domain $D$. The upper limit of the offset $e$ was set to 0.01. Similarly, an initial configuration like that shown in Figure 4(a) was used for all studied cases. The reference domain was chosen according to Figure 3(a).

Four cases were studied in which the output spring stiffness $k_{s}$ was set to $0.5,0.1,0.01$, and $0.005 \mathrm{~N} / \mathrm{m}$. For all studied cases, the maximum number of iterations for the optimization process was 100 . The corresponding topology results of the four studied cases are shown in Figure 8. The results are compared quantitatively in Table 2.

Stiffness can considerably affect the outcome of the optimization process. The spring stiffness must be carefully chosen to obtain a meaningful design.

\subsection{Effect of the Volume Constraint}

We also examined the impact of the volume constraint on the obtained flexure hinges. Two values of the maximum material usage constraint $[\mathrm{Vol}]$ were considered that corresponded to $30 \%$ and $10 \%$ of the whole design domain. The design parameters were set to be the same as those described in Section 5.1. The final designs are shown in Figure 9. The values of $u_{1 x}$ in the two studied cases were $8.3787 \mu \mathrm{m}$ (Figure 9a) and $12.8315 \mu \mathrm{m}$ (Figure 9b). The 


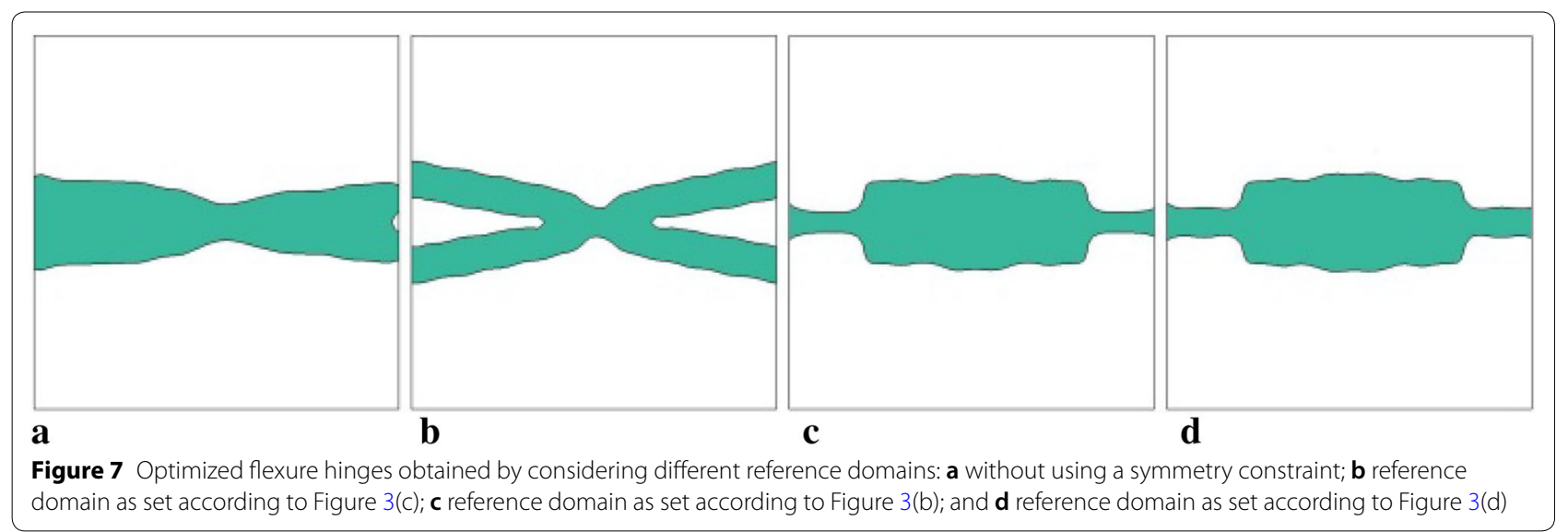

\begin{tabular}{lccc}
$\begin{array}{l}\text { Table 1 Effect of the } \\
\text { on the optimized flexure hinges }\end{array}$ & symmetry & constraint \\
\hline & $\boldsymbol{u}_{\mathbf{1 y}}(\boldsymbol{\mu m})$ & $\boldsymbol{u}_{\mathbf{1 x}}(\boldsymbol{\mu m})$ & $\boldsymbol{e}$ \\
\hline Figure 7(a) & 49.0379 & 10.8671 & 0.002 \\
Figure 7(b) & 49.1271 & 10.1342 & 0.004 \\
Figure 7(c) & 49.5760 & 12.2958 & 0.022 \\
Figure 7(d) & 49.6898 & 12.3232 & 0.021 \\
\hline
\end{tabular}

Table 2 Effect of the spring stiffness $\boldsymbol{k}_{s}$ on the optimized flexure hinges

\begin{tabular}{lrrll}
\hline $\boldsymbol{k}_{\boldsymbol{s}}(\mathrm{N} / \mathrm{m})$ & $\boldsymbol{u}_{\mathbf{1} \boldsymbol{y}}(\boldsymbol{\mu m})$ & $\boldsymbol{u}_{\mathbf{1 x}}(\boldsymbol{\mu m})$ & $\boldsymbol{a}$ & $\boldsymbol{e}$ \\
\hline 0.5 & 1.9977 & 9.5335 & 4.72 & 0.082 \\
0.1 & 9.9624 & 10.5726 & 1.06 & 0.011 \\
0.01 & 98.8749 & 10.9197 & 0.11 & 0.003 \\
0.005 & 198.7659 & 12.3232 & 0.06 & 0.009 \\
\hline
\end{tabular}

offset $e$ of the two cases were 0.006 (Figure 9a) and 0.009 (Figure 9b). These results reveal that the topology of the final designs was affected by changing the maximum material usage.

\subsection{Effect of the Design Domain Configuration}

The aforementioned cases all assumed the design domain to be rectangular. We next examined the optimized configurations of the flexure hinges when a design domain with a different shape or topology was used. Two cases were studied. The design domains are shown in Figure 10.

For the first case, the design domain was actually set to be a right circular flexure hinge. The goal of this design was thus to remove some of the material within

a right circular flexure hinge to obtain a new hinge. As indicated in Ref. [24], although right circular flexure hinges are precise in rotation, their compliance and range of motion are small. Therefore, removing some material from the right circular flexure hinge is helpful to improving its performance. The optimization process is shown in Figure 11. The final topology revealed that the four sharp corners of the design domain were removed. The most important change was that two notches were generated on both sides of the design. With the increase in $k_{s}$, the obtained flexure hinge had a tendency to become two parallel cantilever beams, as indicated in Figure 8(a). This was because when $k_{s}$ is large, $u_{1 x}$ becomes an overwhelming part in

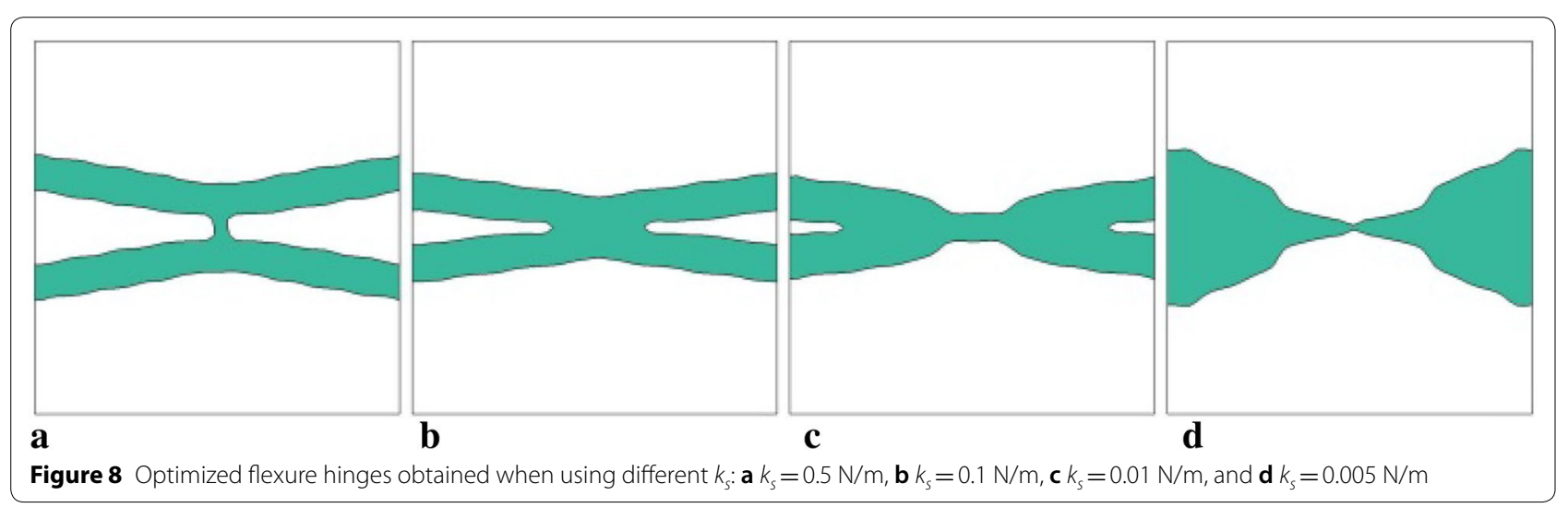



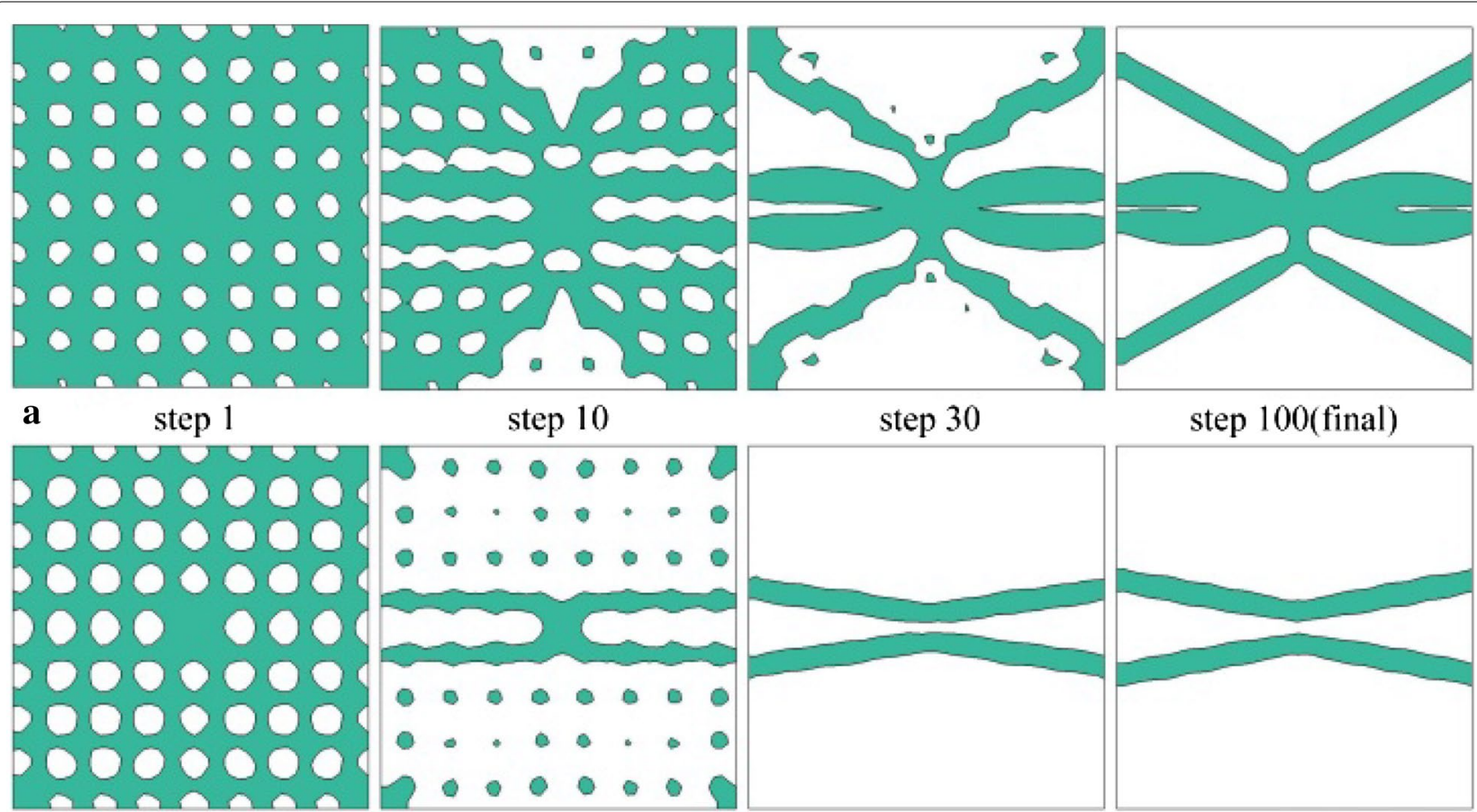

b

step 1

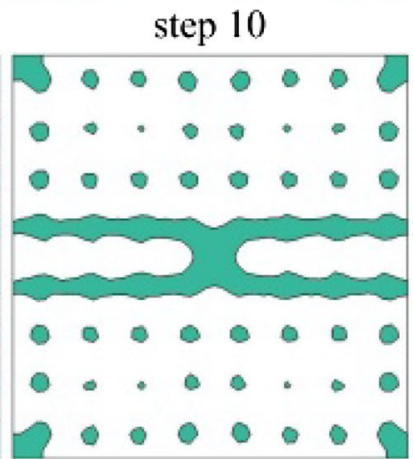

step 10

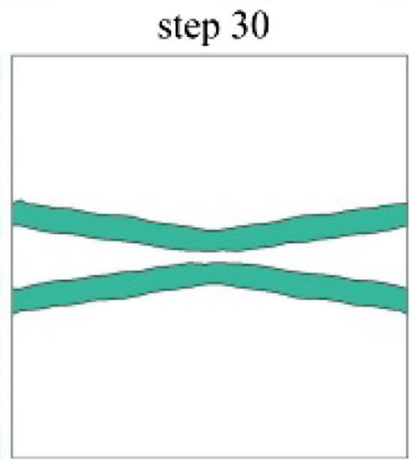

step 30 step 100(final)

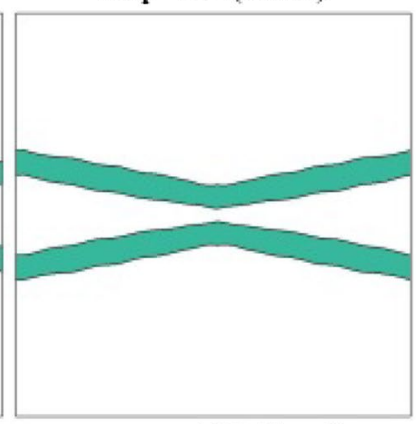

step 100(final)

Figure 9 Optimization processes of the flexure hinges obtained by using different volume constraints: a $0.3, \mathbf{b} 0.1$

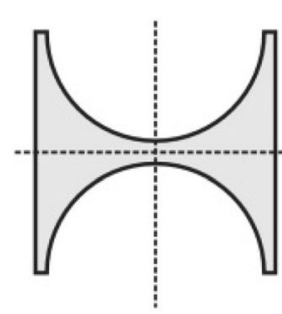

$\mathbf{a}$

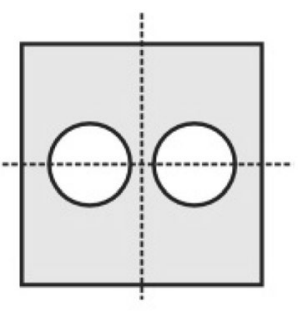

b
Figure 10 Two alternative design domains: $\mathbf{a}$ case 1, and $\mathbf{b}$ case 2

Eq. (5). Therefore, the design problem becomes a problem of minimizing the compliance along the $x$ direction. When the spring stiffness $k_{s}$ is small (e.g., $k_{s}=$ 0.1 or $0.01 \mathrm{~N} / \mathrm{m}$ ), topologies that are similar to that of the cartwheel flexure hinge will be obtained, as shown in Figure 8(b) and (c). When $k_{s}$ is very small, as indicated in Figure 8(d), a notch type flexure hinge will be obtained. In addition, the thickness of the material in the middle of the design domain becomes very thin, which may indicate that this result was not practically applicable. A previous study demonstrated that these types of flexure hinges have better output performance without losing rotational accuracy [24]. For the second case, two holes were inserted inside the rectangular design domain, indicating that these two areas have no material. The optimization process is shown in Figure 12. These results show that the topology of the final designs could be affected by the shape or topology of the design domain.

\section{Conclusions}

A method for topology optimization of single-axis flexure hinges based on the level set method was proposed. A capable optimization model was developed by considering a multi-criteria objective function and a symmetry constraint. Several numerical examples were presented to demonstrate the validity of the proposed method. In addition, the obtained results revealed that the design of a flexure hinge starting from the topology level yielded more choices for compliant mechanism design and obtained better designs that achieved higher performance.

Future studies will consider flexure hinges that are subjected to large deflections, which means that a nonlinear finite element analysis method will be used for elastic 

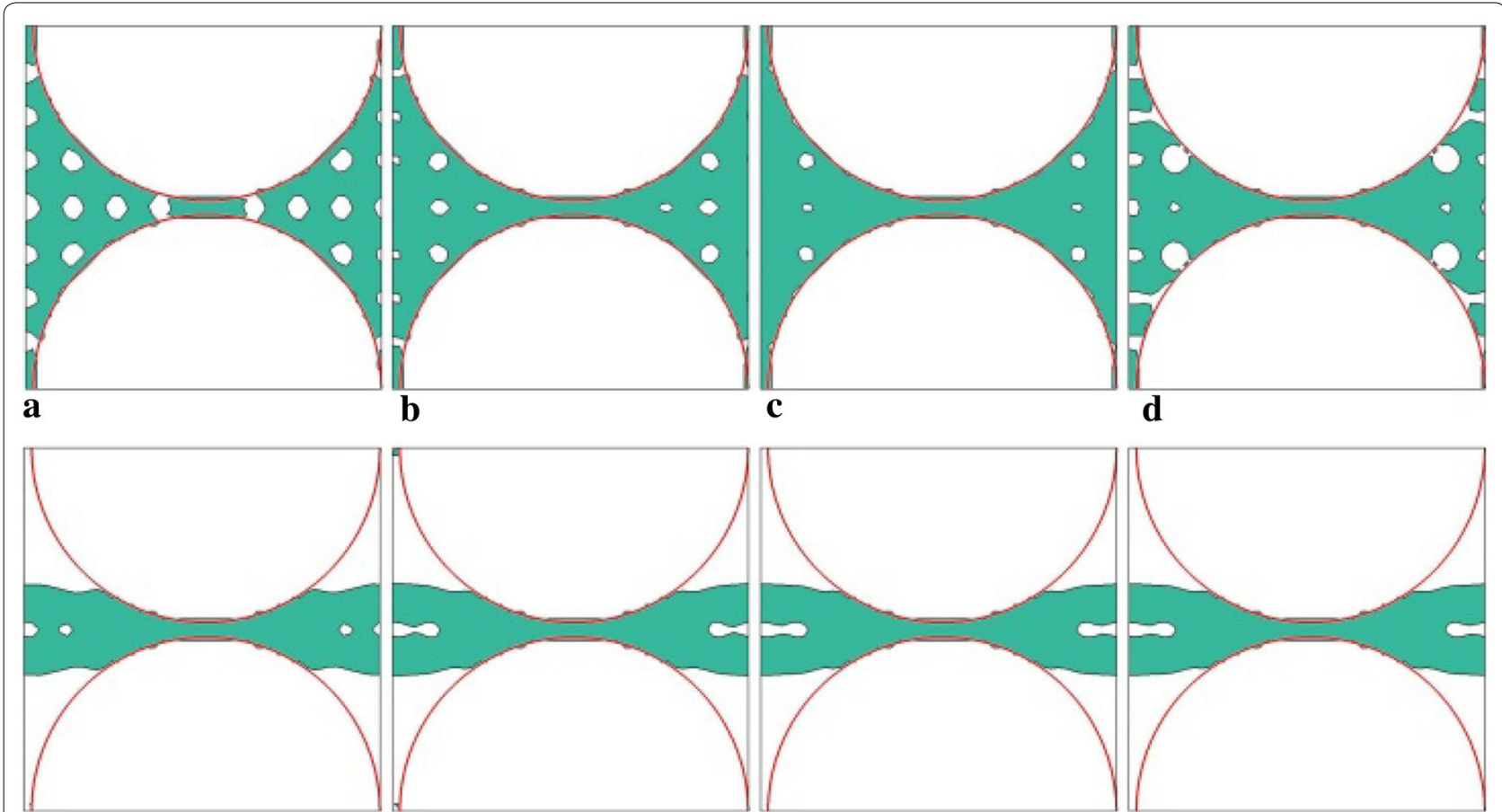

f
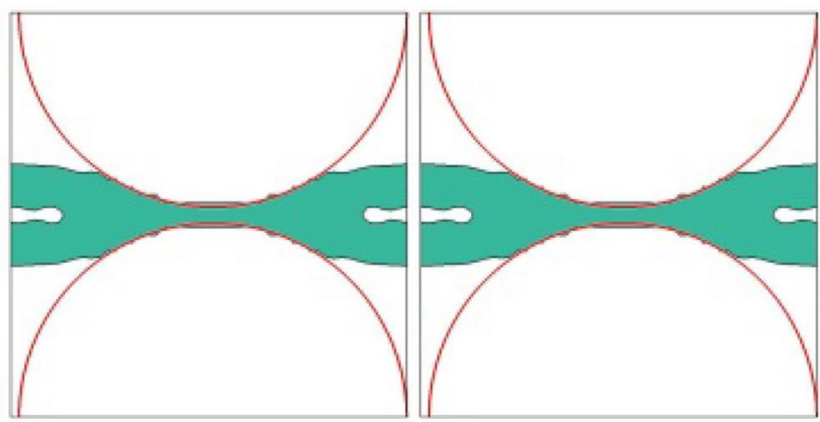

g

h

Figure 11 Optimization process when using the design domain shown in Figure 10(a): a initial configuration, b step 1, c step 5, d step 10, e step 20, f step 40, g step 60, and $\mathbf{h}$ step 100 (final)



Figure 12 Optimization process when using the design domain shown in Figure 10(b): a initial configuration, b step 1, c step 5, d step 10, e step 20, f step 40, g step 60, and $\mathbf{h}$ step 100 (final) 
analysis. Furthermore, the stress concentration problem must be further investigated, as the obtained flexure hinges sometimes have very thin parts that may suffer stress concentration.

\section{Authors' Contributions}

BZ was in charge of the whole trial; BZ and XZ wrote the manuscript; All authors analyzed the data, discussed the results and commented on the manuscript. All authors read and approved the final manuscript.

\section{Author Details}

${ }^{1}$ Guangdong Provincial Key Laboratory of Precision Equipment and Manufacturing Technology, South China University of Technology, Guangzhou 510640, China. ${ }^{2}$ East China Jiaotong University, Nanchang 330013, China.

\section{Authors' Information}

Benliang Zhu, born in 1986, received the Ph.D. degree in mechanical engineering from the South China University of Technology, Guangzhou, China, in 2014. He is currently an associate professor with the South China University of Technology. His research interests include compliant mechanisms, topology optimization, precision positioning, and manipulation in the micro- and nanoscale.

Xianmin Zhang, born in 1964, received the Ph.D. degree in mechanical engineering from Beihang University, Beijing, China, in 1993. He is currently a Professor with the South China University of Technology, Guangzhou, China. He is the Dean of the School of Mechanical and Automotive Engineering, South China University of Technology and the Director of the Guangdong Key Laboratory of Precision Equipment and Manufacturing Technology. His research interests include microelectromechanical system, precision equipment, compliant mechanisms, and advanced manufacturing.

Min Liu, born in 1990, received the Ph.D. degree in mechanical engineering from the South China University of Technology, Guangzhou, China, in 2017. He is currently a Lecturer with the East China Jiaotong University. His research interests include compliant mechanisms, topology optimization and flexure hinges

Qi Chen, born in 1992, is currently a Ph.D candidate at South China University of Technology, Guangzhou, China. His research interests include topology optimization, metamaterial and precision engineering.

Hai Li, born in 1990, is currently a Ph.D candidate at South China University of Technology, Guangzhou, China. His research interests include micro-vision based micro/nano-manipulation and compliant mechanisms.

\section{Competing interests}

The authors declare that they have no competing interests.

\section{Funding}

Supported by National Natural Science Foundation of China (Grant Nos. 51605166, 51820105007), Fundamental Research Funds for the Central Universities of China.

\section{Publisher's Note}

Springer Nature remains neutral with regard to jurisdictional claims in published maps and institutional affiliations.

Received: 30 August 2017 Accepted: 19 February 2019 Published online: 26 February 2019

\section{References}

[1] S Zimmermann, TTiemerding, S Fatikow. Automated robotic manipulation of individual colloidal particles using vision-based control. IEEE/ASME Transactions on Mechatronics, 2015, 20(5): 2031-2038.

[2] H Li, G Hao, R C Kavanagh. A new xyz compliant parallel mechanism for micro-/nano-manipulation: Design and analysis. Micromachines, 2016 7(2): 23.
[3] R Wang, X Zhang. Optimal design of a planar parallel 3-dof nanopositioner with multi-objective. Mechanism and Machine Theory, 2017, 112: 61-83.

[4] H Wu, X Zhang, J Gan, et al. High-quality correspondence imaging based on sorting and compressive sensing technique. Laser Physics Letters, 2016, 13(11): 115205

[5] H Li, X Zhang, HWu, et al. Line-based calibration of a micro-vision motion measurement system. Optics and Lasers in Engineering, 2017, 93: 40-46.

[6] N Lobontiu. Compliant mechanisms: design of flexure hinges. CRC Press, 2002.

[7] L L Howell. Compliant Mechanisms. Springer London, 2013.

[8] M Callegari, A Cammarata, A Gabrielli, et al. Analysis and design of a spherical micromechanism with flexure hinges. Journal of Mechanical Design, 2009, 131(5): 051003.

[9] M Verotti, R Crescenzi, M Balucani, et al. Mems-based conjugate surfaces flexure hinge. Journal of Mechanical Design, 2015, 137(1): 012301.

[10] Y Wu, Z Zhou. Design calculations for flexure hinges. Review of Scientific Instruments, 2002, 73(8): 3101-3106.

[11] G Chen, X Liu, Y Du. Elliptical-arc-fillet flexure hinges: Toward a generalized model for commonly used flexure hinges. Journal of Mechanical Design, 2011, 133(8): 081002.

[12] G Chen, X Liu, H Gao, et al. A generalized model for conic flexure hinges. Review of Scientific Instruments, 2009, 80(5): 055106

[13] ST Smith, V G Badami, J S Dale, et al. Elliptical flexure hinges. Review of Scientific Instruments, 1997, 68(3): 1474-1483.

[14] N Lobontiu. Modeling and design of planar parallel-connection flexible hinges for in- and out-of-plane mechanism applications. Precision Engineering, 2015, 42(1): 113-132.

[15] R Lin, X Zhang, X Long, et al. Hybrid flexure hinges. Review of Scientific Instruments, 2013, 84(8): 394-398.

[16] N Lobontiu, J S Paine, E OMalley, et al. Parabolic and hyperbolic flexure hinges: Flexibility, motion precision and stress characterization based on compliance closed-form equations. Precision Engineering, 2002, 26(2): 183-192.

[17] Y Tian, B Shirinzadeh, D Zhang, et al. Three flexure hinges for compliant mechanism designs based on dimensionless graph analysis. Precision Engineering, 2010, 34(1): 92-100.

[18] J J Yu, X Pei, S S Bi, et al. A unified approach to type synthesis of both rigid and flexure parallel mechanisms. Science in China, 2011, 54(5): 1206-1219.

[19] J B Hopkins, M L Culpepper, Synthesis of multi-degree of freedom, parallel flexure system concepts via Freedom and Constraint Topology (FACT)_Part I: Principles. Precision Engineering, 2010, 34(2): 259-270.

[20] P Xu, J Yu, G Zong, et al. The stiffness model of leaf-type isosceles trapezoidal flexural pivots. Journal of Mechanical Design, 2008, 130(8): 082303.

[21] P Xu. A family of butterfly flexural joints: Q-litf pivots. Journal of Mechanical Design, 2012, 134(12): 121005.

[22] B Zhu, X Zhang, S Fatikow. Design of single-axis flexure hinges using continuum topology optimization method. Science China Technological Sciences, 2014, 57(3): 560-567.

[23] M Liu, X Zhang, S Fatikow. Design of flexure hinges based on stress constrained topology optimization. Proceedings of the Institution of Mechanical Engineers, Part C: Journal of Mechanical Engineering Science, 2016, 231(24): 4635-4645.

[24] M Liu, X Zhang, S Fatikow. Design and analysis of a multi-notched flexure hinge for compliant mechanisms. Precision Engineering, 2017, 48: 292-304.

[25] L Cao, A T Dolovich, W J Zhang. Hybrid compliant mechanism design using a mixed mesh of flexure hinge elements and beam elements through topology optimization. Journal of Mechanical Design, 2015 137(9): 092303.

[26] M P Bendsoe, O Sigmund. Topology optimization: theory, methods, and applications. Springer, 2004.

[27] M P Bendsøe, E Kikuchi. Generating optimal topologies in structural design using a homogenization method. Computer Methods in Applied Mechanics and Engineering, 1988, 71(2): 197-224.

[28] K Maute, A Tkachuk, J Wu, et al. Level set topology optimization of printed active composites. Journal of Mechanical Design, 2015, 137(11): 111402.

[29] B Zhu, X Zhang, Y Zhang, et al. Design of diaphragm structure for piezoresistive pressure sensor using topology optimization. Structural and Multidisciplinary Optimization, 2017, 55(1): 317-329. 
[30] B Zhu, X Zhang, S Fatikow. Level set-based topology optimization of hinge-free compliant mechanisms using a two-step elastic modeling method. Journal of Mechanical Design, 2014, 136(3): 031007.

[31] B S Lazarov, F Wang, O Sigmund. Length scale and manufacturability in density-based topology optimization. Archive of Applied Mechanics, 2016, 86(1-2): 189-218.

[32] M P Bendsøe, O Sigmund. Material interpolation schemes in topology optimization. Archive of Applied Mechanics, 1999, 69(9-10): 635-654.

[33] O Sigmund, P M Clausen. Topology optimization using a mixed formulation: An alternative way to solve pressure load problems. Computer Methods in Applied Mechanics and Engineering, 2007, 196(13-16): 1874-1889.

[34] Y M Xie, X Huang. Recent developments in evolutionary structural optimization (ESO) for continuum structures. IOP Conference Series: Materials Science and Engineering, 2010, 10: 012196.

[35] M Zhou, G I N Rozvany. On the validity of ESO type methods in topology optimization. Structural and Multidisciplinary Optimization, 2001, 21(1): 80-83.

[36] B Zhu, R Wang, H Li, et al. A level set method with a bounded diffusion for structural topology optimization. Journal of Mechanical Design, 2018, 140(7): 071402.

[37] G Allaire, F Jouve, A M Toader. Structural optimization using sensitivity analysis and a level-set method. Journal of Computational Physics, 2004, 194(1): 363-393.
[38] N P V Dijk, K Maute, M Langelaar, et al. Level-set methods for structural topology optimization: a review. Structural and Multidisciplinary Optimization, 2013, 48(3): 437-472.

[39] M Liu, X Zhang, S Fatikow. Design and analysis of a high-accuracy flexure hinge. Review of Scientific Instruments, 2016, 87(5): 055106.

[40] S Osher, R P Fedkiw. Level set methods: An overview and some recent results. Journal of Computational Physics, 2001, 169(2): 463-502.

[41] B Zhu, X Zhang, S Fatikow. Structural topology and shape optimization using a level set method with distance-suppression scheme. Computer Methods in Applied Mechanics and Engineering, 2015, 283: 1214-1239.

[42] Y K Yong, T F Lu, D C Handley. Review of circular flexure hinge design equations and derivation of empirical formulations. Precision Engineering, 2008, 32(2): 63-70

[43] B Zhu, X Zhang, N Wang, et al. Topology optimization of hinge-free compliant mechanisms using level set methods. Engineering Optimization, 2014, 46(5): 580-605.

[44] M Zhou, M. Y Wang. A semi-Lagrangian level set method for structural optimization. Structural and Multidisciplinary Optimization, 2012, 46(4): 487-501.

[45] J Sokolowski, J P Zolesio. Introduction to shape optimization. Springer, 1992

\section{Submit your manuscript to a SpringerOpen ${ }^{\odot}$ journal and benefit from:}

- Convenient online submission

- Rigorous peer review

- Open access: articles freely available online

- High visibility within the field

Retaining the copyright to your article

Submit your next manuscript at $\gg$ springeropen.com 